\title{
Progression of Vertebral Artery Dissection: Vessel Wall Enhancement and Aneurysm Dilation
}

\author{
Song Lee, Hyun Seok Choi, Jinhee Jang, So Lyung Jung, Kook-Jin Ahn, Bum-soo Kim, Jaseong Koo, \\ Yong Sam Shin
}

Keywords: Dissection, Neuroimaging, Vascular, Magnetic resonance imaging

A 35-year-old female with right ptosis and right facial and left limb paraesthesia visited our emergency room. Initial diffusionweighted MRI revealed right lateral medullary infarct. Digital subtraction angiography (DSA) demonstrated dissection of pearland-string type involving a segment including a posterior inferior cerebellar artery (PICA) origin of the right distal vertebral artery. The natural course of unruptured intracranial vertebral artery dissection had been reported to be benign, with a high chance of spontaneous healing. ${ }^{1,2}$ However, in this patient the dissection changed to saccular aneurysm type on follow-up contrast-enhanced MRI and DSA after nine months. The diameter of the dilated segment was also increased from 4 to $8 \mathrm{~mm}$. Furthermore, wall enhancement was noted in the dissected arterial wall. The wall enhancement in the cervical artery dissection has been reported to be suggestive of inflammation and mostly resolve within six months. ${ }^{3}$ Vessel wall enhancement can also be seen in such other arterial diseases as atherosclerosis, moyamoya disease and vasculitis. ${ }^{4}$ Nevertheless, taking account of the change in geometry and the persistent vessel wall enhancement together, we concluded that the dissection had progressed despite conservative treatment.
Thus, stent-assisted coil embolization was performed in the patient without complication.

\section{Disclosures}

SL, HSC, JJ, SLJ, KJA, BK, JK, and YSS hereby declare that they have no conflicts of interest to disclose.

\section{REFERENCES}

1. Mokri B, Houser OW, Sandok BA, Piepgras DG. Spontaneous dissections of the vertebral arteries. Neurology. 1988;38:880-5.

2. Arnold M, Bousser MG, Fahrni G, Fischer U, Georgiadis D, Gandjour J, Benninger $\mathrm{D}$, et al. Vertebral artery dissection: presenting findings and predictors of outcome. Stroke. 2006;37:2499-503. Epub ahead of print Sep 7.

3. Pfefferkorn T, Saam T, Rominger A, Habs M, Gerdes LA, Schmidt C, et al. Vessel wall inflammation in spontaneous cervical artery dissection: a prospective, observational positron emission tomography, computed tomography, and magnetic resonance imaging study. Stroke. 2011;42:1563-8. Epub ahead of print Apr 21.

4. Choi YJ, Jung SC, Lee DH. Vessel wall imaging of the intracranial and cervical carotid arteries. J Stroke. 2015;17:238. Epub ahead of print Sep 30

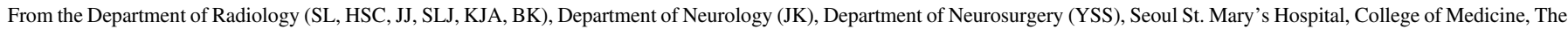
Catholic University of Korea, Seoul, Korea.

Received August 3, 2015. Final Revisions Submitted February 19, 2016. Date of Acceptance March 31, 2016.

Correspondence to: Hyun Seok Choi, Department of Radiology, College of Medicine, The Catholic University of Korea, 11 Jalan Tan Tock, Seoul 137-701, Republic of Korea.

Email: hschoi@catholic.ac.kr 

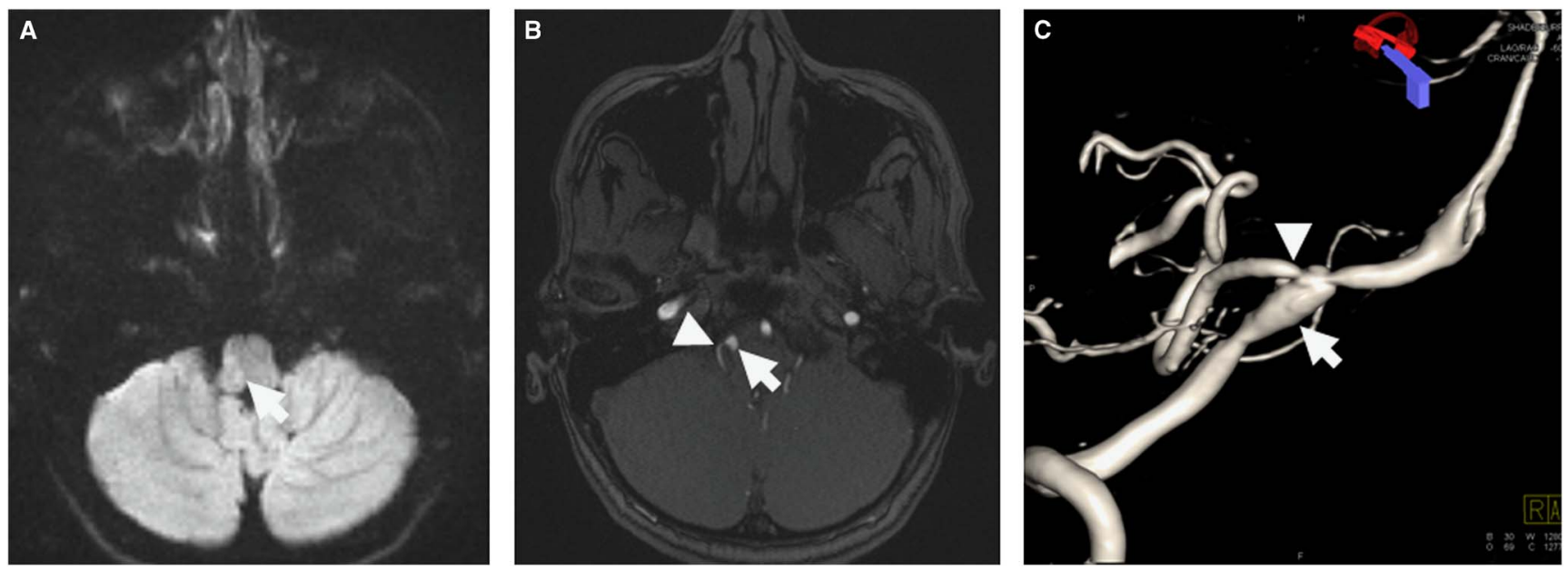

Fig. 1: Initial MRI and DSA. Diffusion-weighted image (A) shows hyperintensity in right lateral medulla, suggestive of acute infarction. Magnetic resonance angiography (MRA) source image $(B)$ and three-dimension $(3 D)$ rotational reconstructed image of DSA (C) show focal stenosis and fusiform dilatation (arrow) at distal vertebral artery, proximally to origin of PICA (arrowhead).
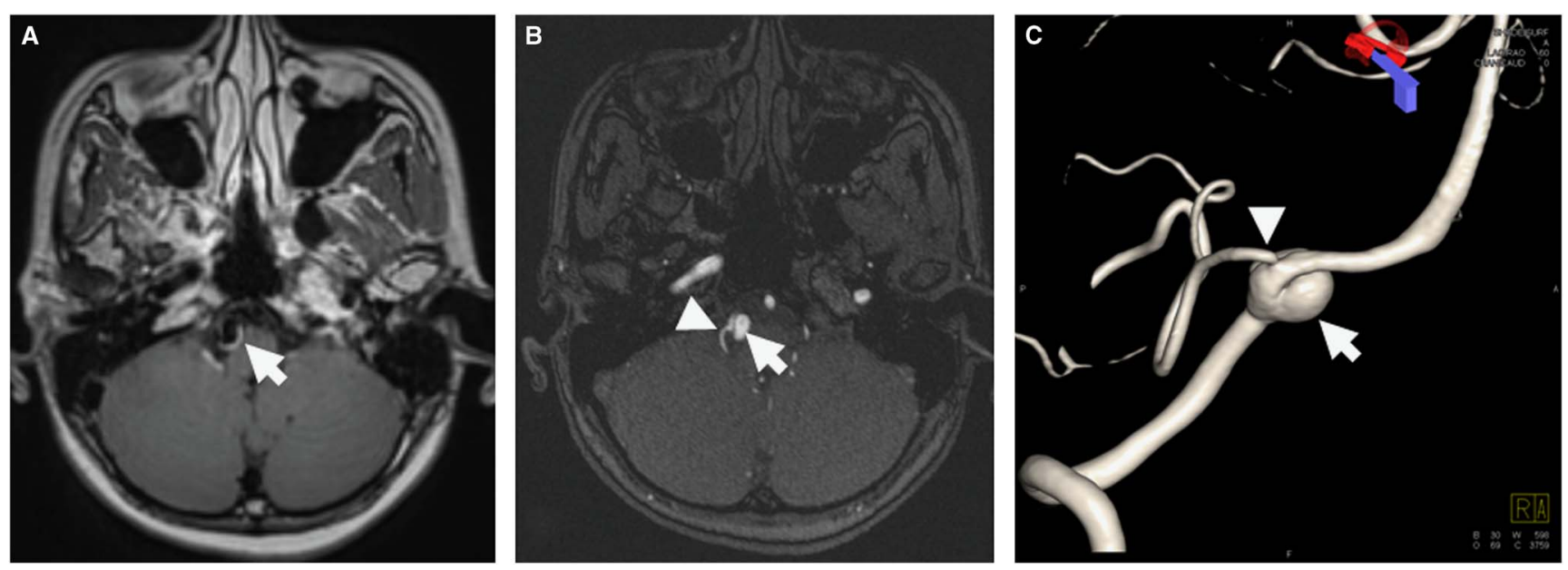

Fig. 2: Six-month follow-up MRI and DSA of the same patient. 3D-SPACE (A) shows wall enhancement (arrow) of dissecting aneurysm. MRA source image $(B)$ and 3D-rotational reconstructed image of DSA $(C)$ show interval change of saccular aneurysm formation at distal vertebral artery (arrow), proximally to PICA origin (arrowhead). 